\title{
A SIMPLE SCHEME FOR ADAPTING TIME-FREQUENCY REPRESENTATIONS
}

\author{
Douglas L. Jones ${ }^{1}$ and Richard G. Baraniuk ${ }^{2}$. \\ ${ }^{1}$ Department of Electrical and Computer Engineering \\ University of Illinois, Urbana, IL 61801 \\ ${ }^{2}$ Laboratoire de Traitement du Signal, Laboratoire de Physique \\ Ecole Normale Supérieure de Lyon \\ 46 allée d'Italie, 69364 Lyon Cedex 07 France
}

\begin{abstract}
Signal-dependent time-frequency representations, in which the kernel or window adapts to the signal being analyzed, perform much better than traditional time-frequency representations for many types of signals. Current signal-dependent time-frequency representations are blockoriented methods suited only for short-duration signals, and many are quite expensive computationally. We propose here a simple, computationally efficient technique for creating adaptive time-frequency representations, in which a kernel characterized by one free parameter is adapted over time. Time adaptation of the kernel allows continuous updating of the kernel to optimally track changes in signal characteristics. The procedure computes a short-time quality measure (concentration or entropy based) of the timefrequency representation for a range of values of the free parameter, and estimates the parameter value maximizing the quality measure via interpolation. Many representations, such as short-time Fourier transforms, cone-kernel distributions, or continuous wavelet transforms, can easily be made adaptive, with a computational cost of the same order as the fixed-kernel representations. Simple examples illustrate the benefits of this technique over fixed-kernel representations.
\end{abstract}

\section{INTRODUCTION}

Time-frequency representations are used in many applications to analyze signals with time-varying frequency content. With the exception of the Wigner distribution, all time-frequency representations employ some kind of smoothing kernel, window, or filter to reduce noise and cross-components. The choice of kernel dramatically affects the appearance and quality of the resulting time-frequency representation. It has been shown that, according to several different measures of performance, the optimal kernel depends on the signal being analyzed [1], [2], [3]. Therefore, the use of any fixed kernel severely limits the class of signals for which the resulting time-frequency representation can perform well.

As an example, consider the signal shown in Fig. 1. It contains several narrow pulses, two sinusoids that overlap

*This work was supported by the Joint Services Electronics Program, Grant No. N00014-90-J-1270, the National Science Foundation, Grant No. MIP 90-12747, and the Sound Group of the Computer-Based Education Research Laboratory at the University of Ilinois. R. Baraniuk is on leave from Rice University, P.O. Box 1892, Houston, Texas 77251-1892, USA. in time, and a Gaussian component. Figure 2(a) shows a short-time Fourier transform (STFT) computed using a narrow Gaussian window. The window matches the pulse components well, but smears the sinusoidal and Gaussian components in the frequency direction. Figure 2(b) shows a STFT computed using a long time window. The window matches the sinusoidal components, but smears the pulse and Gaussian components in the time direction. This illustrates the fundamental tradeoff of the STFT: it is impossible to get both good time and good frequency resolution using a single fixed window.

Due to this fundamental limitation of the STFT, a number of researchers have investigated the use of signaldependent or ad aptive time-frequency representations. One promising technique is an adaptive STFT based on maximizing the local energy concentration in time-frequency [1]. This method performs very well in terms of the appearance and localization of the resulting time-frequency representation; however, it requires about three orders of magnitude more computation than a fixed-window STFT, which precludes its use in many applications. A computationally efficient bilinear signal-dependent time-frequency representation has been developed [2], but it is only suitable for off-line, block analysis of short signals. Thus, a need exists for simple, time-adaptive, computationally-efficient time-frequency representations suitable for real-time, online applications.

In this paper, we present a simple, computationally efficient scheme for adapting a single parameter of a timefrequency distribution (TFD). The method maximizes the short-time concentration of the TFD, and it allows parameter adaptation with time and on-line computation. The method is quite general; for example, it can be used to optimize the instantaneous window length in a STFT, the $\tau$ extent of the cone-kernel distribution [4] or the " $Q$-factor" of the mother wavelet in a continuous wavelet transform [5]. The computational cost is only a few times that of an equivalent fixed-kernel TFD, and as demonstrated below, the performance benefits are often quite substantial.

\section{OPTIMIZING TIME-FREQUENCY CONCENTRATION}

The development of a signal-dependent or adaptive timefrequency representation requires a means of determining an appropriate window or kernel function without extensive a priori knowledge of the signal characteristics. Procedures based on mathematical optimality criteria appear most promising [1], [2], [3]. In these approaches, the signal to analyze is used as an input to an optimization problem 
whose solution is the adaptive window or kernel.

One successful approach, reported in [1], is based on the intuition that a window providing high time-frequency concentration results in good time-frequency localization and resolution. In that paper, the parameters (time width and chirp rate) of a Gaussian STFT window are adapted to maximize a measure of concentration created by dividing the $L^{4}$ norm of the STFT by its $L^{2}$ norm, both computed over a local region in the time-frequency plane. Placing the window parameters in a vector $\underline{p}$ and denoting the STFT itself by $D_{p}(t, \omega)$, the local concentration measure is the defined as the following function of time, frequency, and the parameter vector:

$$
C(t, \omega, \underline{p})=\frac{\iint\left|D_{\underline{p}}(\tau, \Omega) w(\tau-t, \Omega-\omega)\right|^{4} d \tau d \Omega}{\left(\iint\left|D_{\underline{p}}(\tau, \Omega) w(\tau-t, \Omega-\omega)\right|^{2} d \tau d \Omega\right)^{2}} .
$$

Here, $w(\tau, \Omega)$ is a two-dimensional lowpass weighting function centered at $r=\Omega=0$; its purpose is to make the measure "local." To obtain the optimal (time and frequency varying) window parameters $\underline{p}^{\star}(t, \omega)$ for a given signal, the value of $C(t, \omega, \underline{p})$ is maximized with respect to $\underline{p}$ at each time $t$ and frequency $\omega$ :

$$
\underline{p}^{\star}(t, \omega)=\operatorname{argmax}_{\underline{p}} C(t, \omega, \underline{p}) .
$$

The local concentration measure (1) is similar to "kurtosis" in statistics, and similar also to a heuristic definition of entropy used in minimum entropy deconvolution in seismic signal processing [6]. Several other "concentration," "entropy," or "peakiness" measures have been studied in seismic applications [7]; for the purposes of adaptive timefrequency analysis, they yield essentially identical results.

Although concentration-based adaptive time-frequency representations work well, determination of the parameters maximizing the concentration can be very expensive. For example, the concentration measure (1) is a nonlinear function of the window parameters and is not analytically calculable. Thus, expensive nonlinear optimization is required to solve the problem (2). The algorithm in [1] requires at least three orders of magnitude more computation than a fixedwindow STFT to optimize the two window parameters at each time and frequency location. This often precludes the practical use of this technique. We now propose a simpler suboptimal adaptive procedure that allows only time adaptivity of a single parameter and finds only an approximate maximizer of concentration. The advantage of this technique is that it requires only a few times the computation of a fixed-parameter transform.

\section{THE ADAPTIVE SCHEME}

The adaptive technique discussed above is not limited to adapting just STFT parameters; any TFD parameterized by a vector $p$ can be optimized. A simplified concentrationbased adaptive TFD is defined by a simplified version of the optimization problem (1), (2):

$$
p^{\star}(t)=\operatorname{argmax}_{p} C(t, p)
$$

with

$$
C(t, p)=\frac{\iint\left|D_{p}(\tau, \Omega) w(\tau-t)\right|^{4} d \tau d \Omega}{\left(\iint\left|D_{p}(\tau, \Omega) w(\tau-t)\right|^{2} d \tau d \Omega\right)^{2}}
$$

Here, $w(\tau)$ is a one-dimensional lowpass weighting function centered at $r=0$, and $p$ is the single (time-varying) adaptive parameter of the TFD. $C(t, p)$ is a measure of the short-time time-frequency concentration of the TFD with parameter $p$.

Ideally, the optimal TFD parameter $p^{\star}(t)$ at each time $t$ would be selected by computing the short-time concentration $C(t, p)$ as a function of the continuous-valued parameter and choosing the maximizing value. However, determination of the concentration for a large number of parameter values could be quite expensive, because each value of the parameter corresponds to a different manifestation of the TFD. We propose that the short-time concentration be computed only for several discrete values $p_{i}, i=1, \ldots, P$, of the parameter, over a range of values that includes its maximum and minimum acceptable values. The resulting shorttime concentrations $C\left(t, p_{i}\right)$ represent samples of $C(t, p)$, so an estimate of the optimal parameter value $p^{\star}(t)$ can be obtained by interpolating between the samples $C\left(t, p_{i}\right)$ to find the maximizing value of $p$. Fortunately, as a rule the concentration measure is well-behaved and slowly varying with $p$, so $C(t, p)$ can be sampled very coarsely with very little degradation in the final result. Experimentally, sampling as coarsely as once per octave and using quadratic or cubic polynomial interpolation yields excellent results.

Once the optimal parameter value $p^{\star}\left(t_{0}\right)$ is computed at time $t_{0}$, the time slice $D\left(t_{0}, \omega\right)$ of the optimal-parameter TFD can be computed based on that value.

If $P$ complete TFDs had to be stored in order to compute the $C\left(t, p_{i}\right)$ values using (4), the simplicity of the procedure would be overshadowed by extensive memory requirements. Fortunately, all of the information in $D_{p}(t, \omega)$ necessary to compute $C(t, p)$ can be summarized in two one-dimensional functions, obtained as the $L^{4}$ and $L^{2}$ norms of each time slice of the transform

$c_{4}(\tau, p)=\int\left|D_{p}(\tau, \Omega)\right|^{4} d \Omega, \quad c_{2}(\tau, p)=\int\left|D_{p}(\tau, \Omega)\right|^{2} d \Omega$.

Using (5), the short-time concentration measure is computed as

$$
C(t, p)=\frac{\int c_{4}(\tau, p)|w(\tau-t)|^{4} d \tau}{\left(\int c_{2}(\tau, p)|w(\tau-t)|^{2} d \tau\right)^{2}}
$$

If the weighting function $w(r)$ has compact support (as it will in practice), then the integrals in (6) are over finite intervals, and only the portions of $c_{4}(\tau, p)$ and $c_{2}(\tau, p)$ centered around time $t$ need be stored.

The total computational cost of this algorithm is equal to the cost of computing $P$ fixed-parameter TFDs with parameter value $p_{i}$, plus the cost of computing the TFD using the optimal parameter, plus the minor cost of updating and interpolating the short-time concentration measures using (5) and (6). That is, the cost is roughly $P+1$ times the cost of computing a single fixed-parameter TFD, where $P$ is usually between five and ten.

\section{EXAMPLES}

\subsection{Adaptive Short-Time Fourier Transform}

As illustrated earlier in Figs. 1 and 2, the choice of a STFT window greatly affects the resulting time-frequency repre- 
sentation. Figure 3 presents an adaptive-window STFT of the 190-sample signal shown in Fig. 1. The length of a Gaussian window was selected as the adjustable parameter $p$ for the algorithm of Section 3. The short-time concentration (6) for five different window lengths $(p=3,6,12,24,48 \mathrm{sam}$ ples) was computed at each time location, and the optimal window length was interpolated using a cubic polynomial. The length of the weighting window $w$ was set to 61 samples. In comparison to Fig. 2, Fig. 3(b) exhibits the benefits of adaptivity by closely matching the time-duration of the most significant signal component at each time. Figure 3(a) shows the optimal window length as a function of time.

\subsection{Adaptive Cone-Kernel TFD}

Heretofore, we have discussed mainly adaptive STFTs. However, the adaptive scheme proposed above is quite general and applies to any TFD that can be parameterized in terms of a single parameter. An important example is the cone-kernel distribution [4], which exhibits the important property of preservation of outer time support. A ${ }^{{ }^{*} \tau} \tau$ extent" parameter characterizes the cone-kernel proposed in [4]; the quality of the resulting TFD often depends greatly on this parameter. Figures 4(a) and (b) illustrate the conekernel distribution of the signal of Fig. 1 for two choices of $\tau$-extent. Clearly, adaptation of this parameter could lead to improved results.

Figure 5 illustrates an adaptive cone-kernel distribution in which the $r$-extent parameter is adapted to minimize a measure of short-time entropy, which we define as

$$
\begin{aligned}
E(t, p) & =-\iint a(t)^{-1}\left|D_{p}(\tau, \Omega) w(\tau-t)\right|^{r} \\
& \cdot \log \left(a(t)^{-1}\left|D_{p}(\tau, \Omega) w(\tau-t)\right|^{r}\right) d \tau d \Omega .
\end{aligned}
$$

Here, $a(t)$ is a time-varying normalization factor

$$
a(t)=\int|D(\tau, \Omega) w(\tau-t)|^{r} d \tau d \Omega,
$$

and $r>0$ is a constant. The magnitude of $D_{p}(\tau, \Omega)$ is used in the entropy calculation, because the cone-kernel distribution can be negative valued; an alternative would be to minimize using the positive part of $D_{p}(\tau, \Omega)$. The short-time entropy can be computed efficiently in a manner similar to (5), (6). For Fig. 5, six different $\tau$-extents, from $p_{1}=5$ to $p_{6}=128$, were used, and the optimal value was interpolated using a quadratic fit. The kurtosis concentration measure (6) yields almost identical results.

\subsection{Adaptive Wavelet Transform}

As a final example, consider the continuous wavelet transform (CWT) [5]. The CWT is an attractive alternative to the STFT in applications where the durations of the signal components scale inversely with their frequency. Given a bandpass wavelet function $g$ whose Fourier transform is centered at frequency $\omega_{0}$ and has bandwidth $B$, the CWT of a signal $s$ can be computed using

$$
D_{p}(t, \omega)=\int s(u) g^{*}\left(\frac{\omega}{p \omega_{0}}(u-t)\right) d u .
$$

The CWT is a constant- $Q$ time-frequency representation, with the $Q$-factor of the analysis being the center-frequencybandwidth quotient $Q=p \omega_{0} / B$.
The value of the parameter $p$ can have a large effect on the quality of the time-frequency representation of the CWT, because it controls the bandwidth of analysis $(\omega B) /\left(p \omega_{0}\right)$ around each analysis frequency $\omega$. Setting $p$ too small results in excessive smearing of signal components in the frequency direction; setting $p$ too large results in excessive smearing of signal components in the time direction. An optimal time-varying $Q$-factor $p^{\star}(t) \omega_{0} / B$ can be determined by either maximizing the kurtosis measure (6) or minimizing the entropy measure (7).

Clearly, other TFDs that are determined by a single parameter can also be adapted using this method.

\section{CONCLUSIONS}

This paper has presented a simple procedure for the automatic optimization and time adaptation of any timefrequency representation with a window/kernel/wavelet adjustable by a single parameter. Even this modest amount of adaptivity often yields greatly improved results, and in some cases achieves most of the benefits promised by more complicated signal-dependent time-frequency representations. This procedure generally requires only five to ten times the cost of a fixed-parameter distribution and supports on-line, real-time computation. Two or more paramenters can be adapted using a multi-dimensional version of this algorithm, but the cost increases geometrically with the number of parameters. This method greatly extends the range of applications for which adaptive time-frequency representations can be considered.

\section{REFERENCES}

1. D.L. Jones and T.W. Parks, "A High Resolution DataAdaptive Time-Frequency Representation," IEEE Trans. Acoust., Speech, Signal Processing ASSP38(12), pp. 2127-2135, December 1990.

2. R.G. Baraniuk and D.L. Jones, "Optimal Kernels for Time-Frequency Analysis," SPIE 1990 Symposium on Optical and Optoelectronic Applied Science and Engineering, San Diego, California, July 8-13, 1990.

3. R.G. Baraniuk and D.L. Jones, "A radially Gaussian, signal-dependent time-frequency representation", Proceedings of the IEEE International Conference on Acoustics, Speech and Signal Processing, Toronto, 1991, pp. 3181-3184.

4. Y. Zhao, L.E. Atlas and R.J. Marks, "The Use of ConeShape Kernels for Generalized Time-Frequency Representation of Nonstationary Signals," IEEE Trans. Acoust., Speech, Signal Processing ASSP-38, pp. 1084-1091, July 1990 .

5. I. Daubechies, "The Wavelet Transform, Time-Frequency Localization and Signal Analysis," IEEE Transactions on Information Theory, Vol. IT-36, No. 5, pp. 961-1005, September, 1990.

6. R.A. Wiggins, "Minimum Entropy Deconvolution," Geoexploration, Vol. 16, pp. 21-35.

7. D. Donoho, "On Minimum Entropy Deconvolution," in D.F. Findley, ed., Applied Time Series Analysis II, Academic Press, New York, 1981, pp. 565-608. 


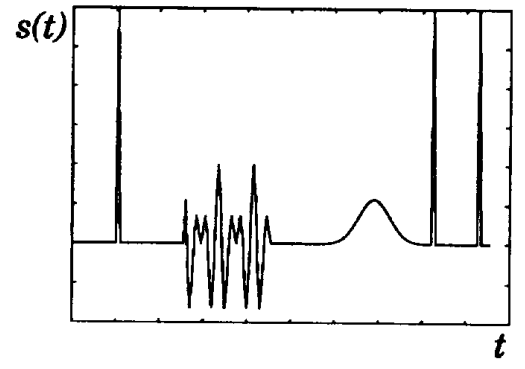

Fig. 1: Test signal.

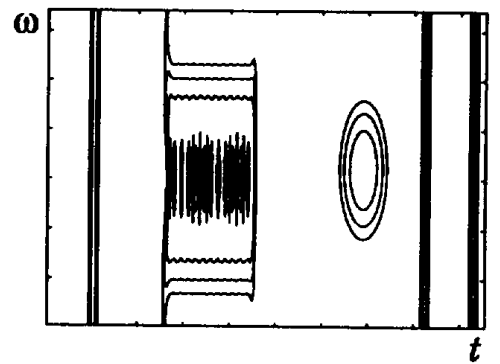

(a)

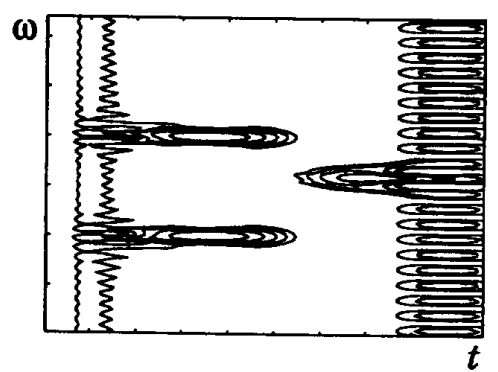

(b)

Fig. 2: STFTs of the test signal computed using (a) a short Gaussian window and (b) a long Gaussian window.

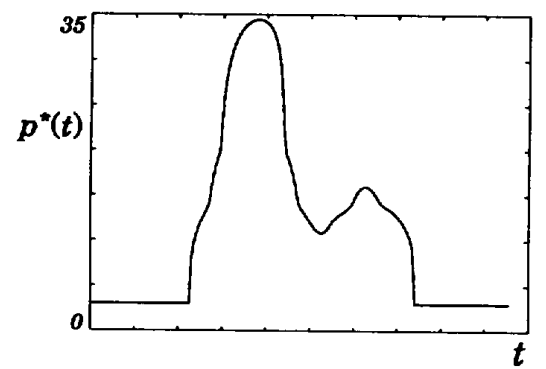

(a)

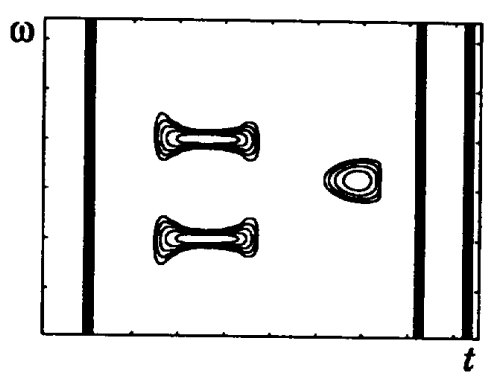

(b)

Fig. 3: (a) Optimal time-varying window length for the STFT of the test signal. (b) Optimal-window STFT.

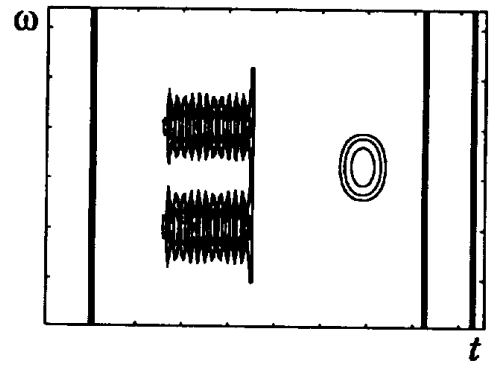

(a)

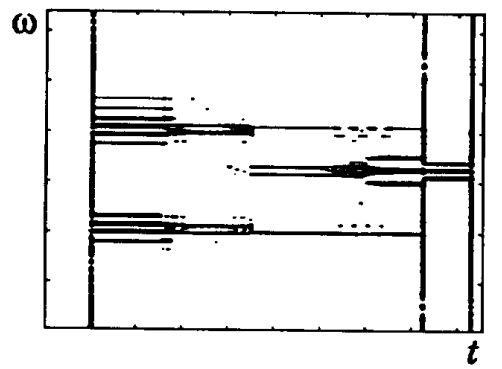

(b)

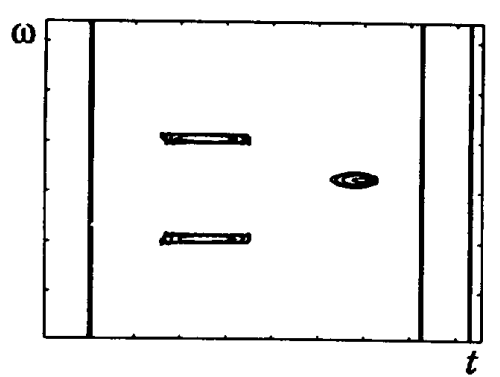

Fig. 4: Cone-kernel distributions of the test signal computed using (a) a small $\tau$-extent parameter and (b) a large $\tau$-extent parameter.

Fig. 5: Cone-kernel distribution using optimal time-varying $\tau$-extent parameter. 